\title{
Incisión de Spangaro en lesiones cardiacas penetrantes, reporte de casos
}

\author{
Spangaro incision in penetrating cardiac lesions, case reports
}

\author{
Mario A. Velázquez-Santiago", José L. Serna-Soto*, Luis R. Meza-López¹, Luis E. Santos-Martínez², \\ Sergio Valladares-Ingram ${ }^{1}$ y Arturo Rueda-Rodríguez ${ }^{1}$ \\ ${ }^{1}$ Servicio de Cirugía General, Hospital General Balbuena, Secretaría de Salud; ${ }^{2}$ Hospital de Cardiología, Unidad Médica de Alta Especialidad, \\ Centro Médico Nacional Siglo XXI, Instituto Mexicano del Seguro Social. Ciudad de México, México
}

\section{Resumen}

Introducción: El trauma en México es un problema mayor de salud pública, siendo una de las principales causas de mortalidad en personas jóvenes. La incidencia reportada de trauma cardiaco varía. El sitio primario de lesión miocárdica es la pared libre del ventrículo derecho. Casos clínicos: Se reportan dos casos de pacientes lesionados con un instrumento punzocortante en el tórax, sufriendo lesión miocárdica que requirió tratamiento quirúrgico de urgencia. Discusión: Existen diversas incisiones del tórax. La elección dependerá de la situación a tratar, el estado hemodinámico y el número de lesiones. Ante una lesión cardiaca, el abordaje de Spangaro es una prudente elección.

Palabras clave: Lesión cardiaca. Taponamiento cardiaco. Toracotomía. Spangaro.

\begin{abstract}
Introduction: Trauma in Mexico is a major public health problem, being one of the main causes of mortality in young people. The reported incidence of cardiac trauma complications. The primary site of myocardial injury is the free wall of the right ventricle. Clinical cases: Two cases of injured patients with a throbbing instrument in the chest are reported, suffering from myocardial injury that required emergency surgical treatment. Discussion: There are various chest incisions. The choice will depend on the entity to be treated, the hemodynamic state, number of injuries. Faced with a heart injury, Spangaro's approach is a prudent choice.
\end{abstract}

Key words: Heart injury. Cardiac tamponade. Thoracotomy. Spangaro.

\section{Introducción}

El trauma en México es un problema mayor de salud pública, siendo una de las principales causas de mortalidad en menores de 45 años ${ }^{1}$. La patología traumática representa el cuarto lugar de muerte en general en México y el primero en edades productivas, de modo que constituye uno de los problemas de salud más grandes, no solo de México², sino del mundo entero ${ }^{3}$; tan solo en 1997 murieron 38 por cada

\section{Correspondencia:}

*José L. Serna-Soto

Cecilio Robelo, s/n, El Parque

Del. Venustiano Carranza

Fecha de recepción: 24-02-2020

C.P. 15970, Ciudad de México, México

E-mail: jlss121289@gmail.com

(http://creativecommons.org/licenses/by-nc-nd/4.0/).
Fecha de aceptación: 26-04-202

DOI: $10.24875 / C I R U .20000137$
Cir Cir. 2020;88(S2):24-30

Contents available at PubMed www.cirugiaycirujanos.com ajo la licencia CC BY-NC-ND 
100,000 personas. En los Estados Unidos mueren al año más de 150,000 personas por trauma, pero son más de 450,000 las que sufren lesiones y todas cursan con algún tipo de incapacidad. El diagnóstico, el tratamiento y la rehabilitación de ese número de lesionados sobrevivientes representa el $40 \%$ del presupuesto que ese país destina a los problemas de salud. Resulta infortunado que en México no conozcamos ese dato 4 .

La incidencia reportada de trauma cardiaco varía mucho: del $8 \%$ en algunas series de autopsias hasta el $76 \%$ en series clínicas. Esta variación se debe a la falta de diagnóstico estandarizado.

El sitio primario de lesión miocárdica es la pared libre del ventrículo derecho, debido a que forma la mayor parte de la superficie anterior del corazón. Las válvulas mitrales y aórticas tienen mayor riesgo de lesiones que las válvulas tricúspides y pulmonares, ya que la presión mural es mayor en lado izquierdo del corazón ${ }^{5}$.

El trauma cardiaco puede clasificarse, según el mecanismo de lesión, en dos grandes categorías: no penetrante (también conocido como lesión cardiaca contundente) y penetrante ${ }^{5}$. El escenario más frecuente de trauma cardiaco penetrante es un joven que se presenta con un apuñalamiento precordial o una herida de bala en el tórax.

La manifestación clínica es comúnmente el taponamiento cardiaco, y dependiendo de la estabilidad hemodinámica del paciente requiere manejo quirúrgico inmediato.

La lesión cardiaca penetrante es una de las causas médicas más letales, con una tasa estimada de mortalidad prehospitalaria del $94 \%$ y una tasa de mortalidad hospitalaria posterior del $50 \%$. Las dos causas más importantes de muerte, como se informa en un estudio de autopsias, son el shock hemorrágico y el taponamiento cardiaco ${ }^{7}$.

El diagnóstico se debe sospechar basándose en la clínica y el interrogatorio; sin embargo, en las lesiones no penetrantes es difícil de hacer, porque los síntomas principales se presentan como los de cualquier trauma torácico no cardiaco.

Los datos de insuficiencia cardiaca congestiva, edema pulmonar, fricción pericárdica o un nuevo soplo cardiaco son menos comunes, pero cuando están presentes debe realizarse una evaluación de lesión cardiaca ${ }^{8}$.

El electrocardiograma se realizará sistemáticamente a todos los pacientes con sospecha de trauma cardiaco. Aunque tiene unas bajas sensibilidad y especificidad cuando se usa solo ${ }^{9}$, los pacientes con hallazgos electrocardiográficos anormales tuvieron complicaciones más significativas que requirieron tratamiento $^{10}$.

Los ensayos de troponina cardiaca de alta sensibilidad con rendimiento analítico mejorado tienen límites de detección más bajos. Sin embargo, incluso estos nuevos ensayos han mostrado reactividad cruzada con el músculo esquelético enfermo ${ }^{11}$. En otras palabras, la troponina cardiaca elevada en un paciente con traumatismo torácico puede ser originada por una lesión del músculo esquelético, y no necesariamente por un problema cardiaco, un trauma o una lesión isquémica asociada.

La ecocardiografía transtorácica es una herramienta muy útil para detectar trauma cardiaco y es de primera elección en pacientes inestables con trauma torácico penetrante o no penetrante.

La tomografía computarizada torácica permite un examen preciso de todo el tórax, incluidos el corazón, el pericardio y los grandes vasos. La tomografía se recomienda como imagen inicial en los pacientes con trauma hemodinámicamente estables ${ }^{12}$.

A continuación, se comentan dos casos de trauma cardiaco penetrante que fueron atendidos por el servicio de cirugía general del Hospital General Balbuena, perteneciente a la red de hospitales de la Secretaría de Salud de la Ciudad de México.

\section{Caso clínico 1}

Varón de 19 años que se presentó, llevado por sus familiares, al servicio de urgencias por traumatismo torácico penetrante, secundario a lesión por objeto punzocortante, de 2 horas de evolución.

Entre sus antecedentes de importancia únicamente destacaban tabaquismo intenso, así como farmacodependencia. A su ingreso refirió dolor precordial, su estado de alerta se encontró alterado con desorientación en espacio, tiempo y persona, además de presentar palidez de tegumentos, diaforesis, mucosas secas y manifestaciones de descarga neurovegetativa (náusea, vómitos y relajación de esfínteres).

A la exploracion física se encontró con presión arterial de 90/60 mmHg, frecuencia cardiaca de 100 latidos por minuto, frecuencia respiratoria de 22 respiraciones por minuto, temperatura de $35.6^{\circ} \mathrm{C}$ y saturación parcial de oxígeno del $83 \%$ al aire ambiente.

A la inspección del tórax anterior se observó una lesión localizada en la línea paraesternal a nivel del quinto espacio intercostal izquierdo de aproximadamente $4 \mathrm{~cm}$, 
con bordes bien definidos y sin evidencia de enfisema ni crepitación. A la auscultación, los ruidos cardiacos eran rítmicos sin agregados, y en el área pulmonar el murmullo vesicular se apreció conservado.

Dentro del abordaje inicial del paciente se procedió a realizar un ultrasonido FAST (Focused Assessment with Sonography for Trauma) y en la ventana pericárdica se apreció una imagen hipoecogénica sugestiva de derrame pericárdico anterior, además de una imagen hiperecogénica hacia el ápex, sugestiva de coágulo, sin trastornos de la movilidad cardiaca.

Se inició reanimación con líquidos y se solicitaron hemoderivados y sala quirúrgica para exploración por probable lesión cardiaca. Durante ese periodo se realizó un nuevo ultrasonido FAST y se apreció un incremento del derrame pericárdico, además de hipocinesia cardiaca generalizada, con colapso de las cavidades derechas, lo cual se correlacionó clínicamente con deterioro hemodinámico y tríada de Beck.

En el quirófano se optó para la exploración quirúrgica por un abordaje mediante toracotomía anterolateral izquierda (Spangaro) (Fig. 1), encontrándose hemotórax izquierdo de aproximadamente $2000 \mathrm{ml}$, además de lesión en el pericardio con comunicación a la pleura izquierda y a través de esta presentaba sangrado profuso. Se procedió a realizar pericardiotomía, localizando una lesión en la cara anterior del ventrículo izquierdo de $2.5 \mathrm{~cm}$ de longitud y bordes irregulares, que correspondió al grado III de la American Association for the Surgery of Trauma. Después del control de la hemorragia mediante digitopresión, se procedió a la reparación quirúrgica con puntos en $\mathrm{U}$ con $\mathrm{Vicryl}^{\circledR}$ 2-0 y un punto simple con seda 3-0 (Fig. 2). Se realizó lavado de cavidad y retiro de coágulos residuales. Durante la cirugía se llevó a cabo reposición de volumen con cuatro paquetes de concentrados eritrocitarios y dos de plasma fresco congelado, una unidad de almidón y Gelafundin ${ }^{\circledR} 4 \%$ (gelatina succinilada). Finalmente, se colocó una sonda pleural de $36 \mathrm{Fr}$.

El paciente evolucionó de forma favorable, hacia el tercer día del posoperatorio se retiraron los drenajes y egresó del hospital al séptimo día de la intervención.

\section{Caso clínico 2}

Varón de 31 años que ingresó al servicio de urgencias 30 minutos despues de ser agredido en la vía pública. Los familiares refirieron que el paciente había recibido una herida por objeto punzocortante en la cara anterior del tórax.

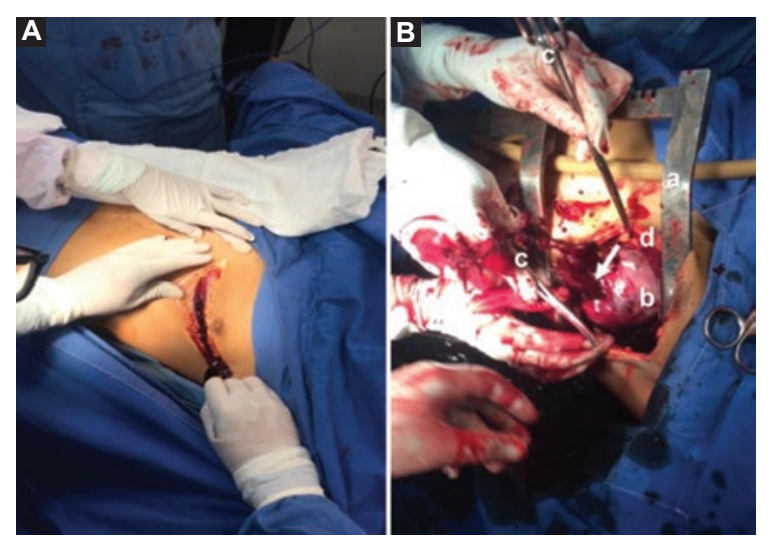

Figura 1. A: abordaje quirúrgico mediante toracotomía anterolateral izquierda (Spangaro). Entre sus principales ventajas está la velocidad con la que puede realizarse para tener rápido acceso a los órganos torácicos. B: tras la apertura del pericardio (tomado entre dos pinzas Allis) puede observarse una lesión en el ventrículo izquierdo. Nótese la abundante salida de sangre (flecha) secundaria a la lesión. a: separador de Finochietto; b: ventrículo izquierdo; c: pinzas Allis; $d$ : pericardio.


Figura 2. A: control del sangrado de la lesión ventricular izquierda mediante digitopresión (flecha), a la vez que se realiza reparación y cardiorrafia ventricular. Se hace énfasis en la exposición de los órganos torácicos que se obtiene mediante la incisión de Spangaro. a: cardiorrafia ventricular; $b$ : separador de Finochietto; c: pericardio; $d$ : pulmón izquierdo; e: pinza de Allis; f: portaagujas Mayo-Hegar. B: ventrículo izquierdo ya reparado (flecha). Se prefiere utilizar polipropileno (Prolene ${ }^{\circledR}$ ) para este tipo de reparaciones. Sin embargo, en casos de urgencia puede utilizarse seda o incluso Vicry ${ }^{\circledR}$ (poliglactina 910). Debe considerarse que la aguja del material de sutura sea de tipo atraumático. a: separador de Finochietto; b: pinza de Allis; c: pericardio; VI: ventrículo izquierdo.

El paciente ingresó al servicio de urgencias con 5 puntos en la Escala de coma de Glasgow y palidez franca de piel y mucosas, por lo que se decidió el manejo avanzado de la vía aérea y reanimación hídrica vigorosa. La presión arterial era de 60/40 mmHg, la frecuencia cardiaca no audible, la temperatura de 


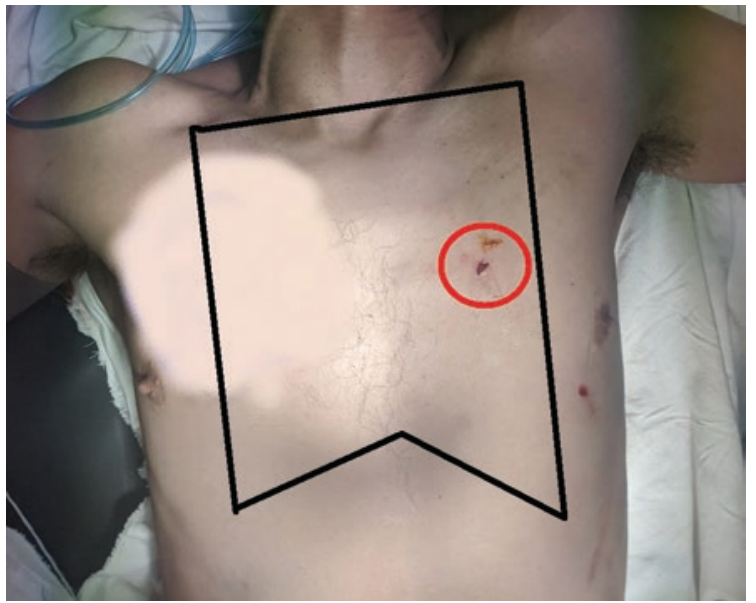

Figura 3. Lesión en la cara anterior del tórax, en la zona conocida como "área peligrosa de Murdock y Sawers", cuyos límites son las clavículas, la línea media clavicular, el reborde costal y la apófisis xifoides. La circunferencia en rojo indica el sitio de la lesión.

$35.6^{\circ} \mathrm{C}$ y la saturación parcial de oxígeno del $83 \%$. Se observó franca ingurgitación yugular y, a la inspección del tórax, una lesión localizada en la línea paraesternal izquierda a nivel del cuarto espacio intercostal de aproximadamente $2 \mathrm{~cm}$ de longitud (Fig. 3).

Debido a la inestabilidad hemodinámica del paciente y a los hallazgos clínicos sugerentes de lesión cardiaca con taponamiento cardiaco, se pasó a quirófano para recibir tratamiento quirúrgico inmediato.

Se inició el abordaje mediante toracotomía anterolateral izquierda (Spangaro). Se encontró el espacio pericárdico ocupado por sangre, por lo que se procedió a realizar pericardiotomía, drenando aproximadamente $300 \mathrm{ml}$ de contenido hemático. Se localizó una lesión en la pared libre del ventrículo derecho de 2 $\mathrm{cm}$ de longitud. Se cerró el defecto mediante punto en $U$ con Vicry ${ }^{\circledR} 1$, se realizó el secado de la cavidad torácica y se colocó una sonda pleural de $36 \mathrm{Fr}$ (Figs. 4 y 5).

El paciente evolucionó favorablemente, se retiraron los drenajes al tercer día y egresó del servicio a los 6 días.

\section{Discusión}

Las lesiones cardiacas penetrantes constituyen una emergencia quirúrgica ${ }^{13,14}$. Cuando se presentan, el tratamiento oportuno en los pacientes que sobreviven al llegar al hospital resulta crítico, y por este motivo, la habilidad para manejar este tipo de situaciones se considera como un indicador para evaluar la calidad



Figura 4. Toracotomía anterolateral izquierda. Obsérvese la cantidad de espacio que se obtiene al abordar lesiones cardiacas. A: separador de Finochietto. B: pulmón izquierdo; flecha: lesión en la pared libre del ventrículo derecho.

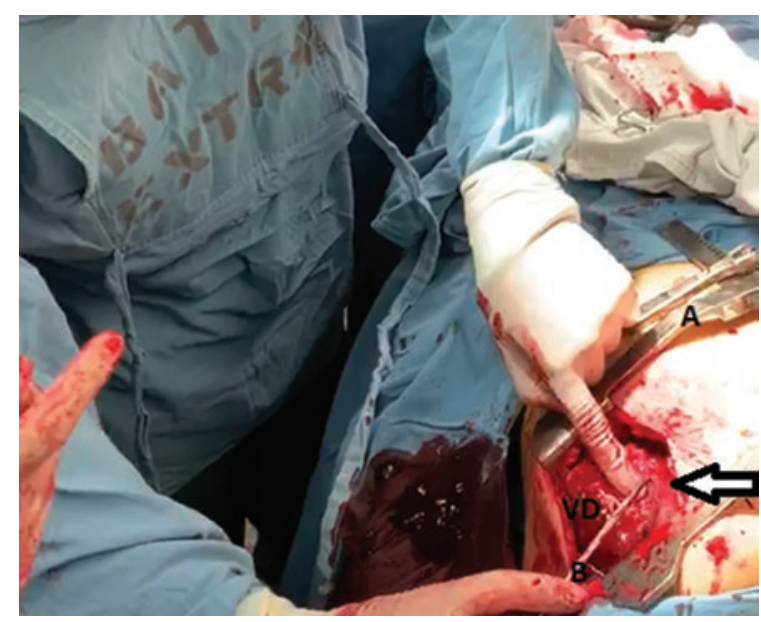

Figura 5. Reparación del ventrículo derecho mediante punto en $U$ con poliglactina 910. A: separador de Finochietto. B: portaagujas MayoHegar. VD: ventrículo derecho; flecha: control digital de la lesión previo a la aplicación de la sutura.

de la atención en los centros de trauma ${ }^{15}$. Aun así, a pesar de los avances en la atención de estos pacientes, la mortalidad continúa siendo elevada, sobre todo en aquellos centros con bajo volumen, debido, entre otros aspectos, al elevado porcentaje de pacientes que fallecen al llegar a los servicios de urgencias y también a protocolos de atención que no reflejan los principios de tratamiento actual en trauma ${ }^{15}$.

Durante la evaluación inicial es necesario reconocer el taponamiento cardiaco, debido a que en la mayoría de los casos es esta la lesión que lleva a la muerte a los pacientes ${ }^{16}$. Para poder comprender su 
fisiopatogenia, hay que recordar que el pericardio es capaz de acomodar la acumulación gradual de sangre si la hemorragia no es lo suficientemente rápida como para causar incrementos agudos de la presión intrapericárdica. Una hemorragia lenta y paulatina es generalmente bien tolerada, debido a que permite cierto grado de adaptación del pericardio. También se debe considerar que el taponamiento cardiaco puede tener un efecto protector, al limitar y contener la hemorragia, y por otro lado un efecto deletéreo, al conducir al paro cardiaco ${ }^{17}$.

En el primer caso aquí reportado, la comunicación entre el pericardio y el hemitórax izquierdo permitió contener la hemorragia cardiaca, a la vez que evitó el taponamiento cardiaco. Si bien el diagnóstico de los pacientes con lesión cardiaca por trauma penetrante se sospecha por el cuadro clínico, las herramientas de diagnóstico, como el ultrasonido $\mathrm{FAST}^{18} \mathrm{y}$ la ventana pericárdica subxifoidea, han incrementado y confirmado tal diagnóstico ${ }^{18-21}$.

Respecto al abordaje quirúrgico, podemos dividir las incisiones torácicas en tres grandes tipos: abordajes abiertos en decúbito lateral (con preservación y sin preservación muscular), abordajes abiertos en posición supina (esternotomía, toracotomía anterior, clamshell y sus variantes), y abordajes híbridos (combinación de abordajes abiertos y mínimamente invasivos ${ }^{22}$.

Los abordajes en decúbito lateral se emplean generalmente para la cirugía pleuropulmonar de tipo electivo. Respecto a la cirugía traumatológica de urgencia y con sospecha de lesión cardiaca, se prefiere el uso de abordajes anteriores (pues permiten una mejor exposición de las lesiones, además de que no existe el riesgo de colapso hemodinámico en caso de cambio de posición), como son la esternotomía media, la toracotomía anterolateral izquierda y la incisión clamshell.

Cada incisión tiene sus ventajas y desventajas, y la elección puede verse influenciada por la experiencia y la familiaridad del cirujano, además de por los recursos del hospital donde se labore ${ }^{23}$. Esta última situación resulta muy importante porque la infraestructura médica necesaria para resolver este tipo de lesiones puede ser insuficiente (falta de instrumental necesario, personal capacitado) o incluso inexistente ${ }^{24}$. Además, la mayoría de los cirujanos generales no cuentan con experiencia en cirugía vascular o en el manejo de los grandes vasos. Dicha experiencia es difícil de adquirir, pero el cirujano general debe estar familiarizado para la atención inicial y la realización de procedimientos. Por lo tanto, es importante que el cirujano general se sienta cómodo con un abordaje que sea rápido y efectivo para operar a aquellos pacientes que no responden a la reanimación inicial y que se encuentran hemodinámicamente inestables y con sospecha de trauma cardiaco ${ }^{24}$.

Por otra parte, las incisiones y los abordajes quirúrgicos varían entre las instituciones ${ }^{25}$. Las numerosas publicaciones acerca de los diversos manejos y abordajes de estas lesiones demuestran que no existe un consenso. Existen estudios que muestran tasas de supervivencia significativas en pacientes a quienes se realizó toracotomía anterolateral para lesiones cardiacas y que fueron admitidos al servicio de urgencias, mientras que otros argumentan que este tipo de abordaje solo debe ser realizado por equipos con la suficiente experiencia para obtener los mejores resultados ${ }^{25}$. Flaris, et al. ${ }^{26}$ subrayan que la incisión tipo clamshell es rápida y no causa pérdida de tiempo. Sin embargo, una pregunta aparte se refiere a qué incisión proporciona el acceso más fácil a la lesión. Besir, et al. ${ }^{25}$ compararon la toracotomía y la esternotomía en pacientes con trauma, y concluyen que ambos abordajes pueden utilizarse de manera complementaria.

La esternotomía media es la incisión de elección en pacientes ingresados con heridas precordiales penetrantes que pueden albergar lesiones cardiacas ocultas o sin compromiso hemodinámico, además de que constituye el método de referencia para los procedimientos de cirugía cardiaca ${ }^{23}$. Su principal ventaja es la gran exposición de las estructuras mediastinales, el control de los vasos principales pulmonares y la posibilidad de abordar ambas cavidades pleurales. Por otra parte, su gran desventaja es la pobre exposición del mediastino posterior, el difícil acceso al lóbulo pulmonar inferior izquierdo, las complicaciones infecciosas como la mediastinitis, y que requiere cierto grado de entrenamiento y equipo para poder llevarse a cabo de manera rápida y efectiva ${ }^{22}$.

Los pacientes admitidos con estabilidad hemodinámica pueden someterse a una investigación preoperatoria limitada con radiografía de tórax 0 ecocardiografía ${ }^{23}$. Del mismo modo, los pacientes que llegan a la sala de operaciones con algún grado de estabilidad pueden someterse a una ventana pericárdica subxifoidea que puede convertirse a esternotomía en caso de confirmación de la lesión ${ }^{23}$. Cuando se encuentra disponible, el uso de una sierra esternal permite que la esternotomía se realice de manera rápida y efectiva, logrando una gran exposición del corazón y los grandes vasos, y además resulta menos dolorosa en comparación con la toracotomía, lo que 
permite una mínima depresión de la función respiratoria. Por otra parte, la esternotomía puede resultar poco ventajosa en caso de que se presenten lesiones concomitantes de la aorta descendente o del esófago, llegando a ameritar incluso el uso de derivación cardiopulmonar, la cual no se encuentra disponible en la mayoría de nuestros hospitales ${ }^{25}$.

Otras desventajas son la pobre exposición del mediastino posterior y el difícil acceso al lóbulo pulmonar inferior izquierdo, lo que la vuelve poco útil en lesiones complejas. Finalmente, deben considerarse las complicaciones infecciosas, como la mediastinitis, y el hecho de que requiere cierto grado de entrenamiento y equipo para poder llevarse a cabo de manera rápida y efectiva ${ }^{22}$.

La toracoesternotomía (clamshell o hemi-clamshell) se considera la incisión estándar para el trasplante pulmonar bilateral. En la actualidad, permanece como una herramienta valiosa en los casos de trauma que ameriten manejo de ambos hemitórax. El abordaje se realiza a través de una incisión a lo largo del pliegue inframamario bilateralmente, y luego se identifica el quinto espacio intercostal y se accede a la cavidad. Ambas arterias mamarias son ligadas y seccionadas, al igual que el cuerpo esternal de manera transversal. La variante hemi-clamshell se realiza mediante una incisión que va desde el manubrio esternal, en la línea media, y después pasando por el pliegue inframamario, hasta la línea axilar anterior. En este caso, el abordaje puede extenderse superiormente hacia el cuello, sobre el borde medial del músculo esternocleidomastoideo (abordaje de Grunenwald) o seccionando la clavícula (abordaje de Dartavelle). Estos abordajes son útiles en el tratamiento de grandes tumores mediastinales, en el trasplante pulmonar bilateral y en caso de trauma. Sin embargo, su uso hoy en día es limitado por sus complicaciones. La división transesternal se acompaña de complicaciones hasta en el $30 \%$ de los casos, en comparación con el 1-2\% con la esternotomía media. Asimismo, el dolor y la deformidad posoperatoria son mayores, con un impacto deletéreo en la mecánica ventilatoria ${ }^{22}$.

Otro abordaje utilizado es la toracotomía anterolateral izquierda (incisión de Spangaro), sobre todo en aquellos pacientes con lesiones cardiacas penetrantes que llegan graves y con inestabilidad hemodinámica al servicio de urgencias, y se requiere explorar el área afectada directamente con el fin de reparar el sitio de sangrado. Además, esta incisión puede extenderse a través del esternón de forma bilateral (abordaje clamshell) en caso de que las lesiones del paciente involucren el hemitórax derecho, permitiendo así la exposición completa del mediastino anterior y ambos hemitórax ${ }^{15,18,21}$.

Como podemos ver, existen diversas variantes para acceder al tórax. La elección dependerá del diagnóstico, si el paciente se encuentra estable o inestable, si es lesión única o múltiple, si hay lesiones asociadas, equipo de cirugía cardiovascular o de cirugía general, y si se cuenta con el material necesario para la intervención. Ante las limitaciones que podría haber en los diferentes rubros mencionados, en caso de una lesión cardiaca el abordaje de Spangaro podría ser una prudente elección.

En cuanto a la reparación quirúrgica, las heridas ventriculares pueden ser reparadas primero mediante la oclusión digital de la laceración mientras se coloca una sutura de colchonero simple o interrumpida horizontal de Halsted ${ }^{13}$. También pueden ser reparadas mediante sutura con monofilamento de Prolene ${ }^{\circledR}$ 2-0. Reparar lesiones cardiacas de heridas penetrantes es menos difícil que reparar lesiones por proyectil de arma de fuego, ya que estas últimas tienden a producir algún grado de defecto por la explosión que causa dificultades al llevar a cabo la reparación ${ }^{13,14}$. Las lesiones por proyectil de arma de fuego que se han suturado y controlado inicialmente se extienden con frecuencia a medida que el miocardio dañado continúa contrayéndose y se vuelve más débil y friable debido al efecto de la ráfaga. Con frecuencia, estas lesiones requieren suturas múltiples en un intento desesperado de controlar la hemorragia masiva18,19,21,23.

Finalmente, a medida que la violencia ha aumentado, muchos de los pacientes llegan con lesiones complejas asociadas. Se ha definido como lesión cardiaca compleja o combinada si se asocia con una lesión vascular cervical, torácica o abdominal, o incluso una lesión vascular periférica, lo que implica un mayor grado de complejidad para su tratamiento. Debe darse prioridad a la lesión que causa la mayor pérdida de sangre, y aquí el desafío consistirá en planear y decidir de forma adecuada el abordaje quirúrgico ${ }^{18,23,27}$.

\section{Agradecimientos}

Los autores agradecen al Dr. Gabriel Mejía Consuelos y al Dr. Francisco Carballo Cruz el apoyo incondicional hacia el equipo de trabajo.

\section{Conflicto de intereses}

Los autores declaran no tener conflicto de intereses. 


\section{Financiamiento}

Los autores declaran que no hubo ninguna clase de patrocinios para el desarrollo de este artículo.

\section{Responsabilidades éticas}

Protección de personas y animales. Los autores declaran que para esta investigación no se han realizado experimentos en seres humanos ni en animales.

Confidencialidad de los datos. Los autores declaran que han seguido los protocolos de su centro de trabajo sobre la publicación de datos de pacientes.

Derecho a la privacidad y consentimiento informado. Los autores han obtenido el consentimiento informado de los pacientes y/o sujetos referidos en el artículo. Este documento obra en poder del autor de correspondencia.

\section{Bibliografía}

1. Yousef R, Carr JA. Blunt cardiac trauma: a review of the current knowledge and management. Ann Thorac Surg. 2014;98:1134-40.

2. Lara AJ, Basilio OA, Arévalo TM. Epidemiología del trauma en México. 2008-2013. Rev Trauma en América Latina. 2015;15(1).

3. Mattox KL, Feliciano DV, Moore EE. Trauma. 7th ed. New York: McGraw Hill Interamericana; 2013.

4. Díaz MA, Basilio OA, Cruz VF, Briones GJ. Trauma, problema de salud en México. Conacyt, Academia Mexicana de Medicina; 2016.

5. Leite L, Gonçalves L, Nuno Vieira D. Cardiac injuries caused by trauma: review and case reports. J Forensic Leg Med. 2017;52:30-4.

6. Campbell NC, Thomson SR, Muckart DJ, Meumann CM, Van Middelkoop I, Botha JB. Review of 1198 cases of penetrating cardiac trauma. $\mathrm{Br} \mathrm{J}$ Surg. 1997;84:1737-40.

7. Altun G, Altun A, Yilmaz A. Hemopericardium-related fatalities: a 10-year medicolegal autopsy experience. Cardiology. 2005;104:133-7.

8. El-Chami MF, Nicholson W, Helmy T. Blunt cardiac trauma. J Emerg Med. 2008;35:127-33.
9. Emet M, Akoz A, Aslan S, Saritas A, Cakir Z, Acemoglu H. Assessment of cardiac injury in patients with blunt chest trauma. Eur J Trauma Emerg Surg. 2010;36:441-7.

10. Maenza RL, Seaberg D, D'Amico F. A meta-analysis of blunt cardiac trauma: ending myocardial confusion. Am J Emerg Med. 1996;14:237-41.

11. Jaffe AS, Vasile VC, Milone M, Saenger AK, Olson KN, Apple FS. Diseased skeletal muscle: a noncardiac source of increased circulating concentrations of cardiac troponin T. J Am Coll Cardiol. 2011:58:1819-24.

12. Restrepo CS, Gutiérrez FR, Marmol-Vélez JA, Ocazionez D, Martínez-Jiménez S. Imaging patients with cardiac trauma. Radiographics. 2012;32:633-49.

13. Asensio JA, Navarro-Soto S, Forno W, Roldan G, Petrone P, Salim A, et al. Penetrating cardiac injuries: a complex challenge. Int J Care Injured. 2001;32:533-43.

14. Asensio JA, O'Shanahan G, Petrone P, Costa D, Robin-Lersundi AJ, Kimbrell BJ. Toracotomía de emergencia: una evaluación critica de la técnica. Cir Esp. 2004;75:171-8.

15. Mina MJ, Rashi-Jhunjhunwala BA, Gelbard RB, Dougherty SD, Carr JS, Dente $\mathrm{CJ}$, et al. Factors affecting mortality after penetrating cardiac injuries: 10-year experience at urban level I trauma center. American $\mathrm{J}$ Surg. 2017;213:1109-15.

16. Janati M, Bolandparvaz S, Salaminia S, Johari HG, Sabet B, Kojuri J. Outcome of penetrating cardiac injuries in southern Iran, Shiraz. Chin J Traumatol. 2013;16:89-93.

17. Asensio JA, Petrone P, Kimbrell B, Kuncir E. Toracotomía de emergencia. Evaluación crítica de la técnica. Rev Colomb Cir. 2006;21:75-86.

18. Kong VY, Oosthuizen G, Sartorius B, Bruce J, Clarke DL. Penetrating cardiac injuries and the evolving management algorithm in the current era. J Surg Res. 2015; 193:926-32.

19. Pereira BM, Nogueira VB, Calderan TR, Villaca MP, Petrucci $O$, Fraga GP. Penetrating cardiac trauma: 20 -y experience from a university teaching hospital. J Surg Res. 2013;183:792-7.

20. Mishra B, Gupta A, Sagar S, Singhal M, Kumar S. Traumatic cardiac injury: experience from a level-1 trauma center. Chin J Traumatol. 2016;19:333-6.

21. Marelli D, Pisano OE, Calafell LA, Herrera FN. Herida cardíaca por arma de fuego. Presentación clínica y conducta terapéutica. Rev Fed Arg Cardiol. 2013;43:45-8.

22. Martin-Ucar A, Socci L. Thoracic incisions for open surgery. Shanghai Chest. 2017;1:1-13.

23. O'Connor J, Ditillo M, Scalea T. Penetrating cardiac injury. J R Army Med Corps. 2009:155:185-90.

24. Doll D, Eichler M, Vassiliu P. Penetrating thoracic trauma patients with gross physiological derangement: a responsibility for the general surgeon in the absence of trauma or cardiothoracic surgeon? World J Surg. 2017:41:170-5.

25. Besir Y, Gökalp O, Eygi B. Choice of incision in penetrating cardiac injuries: which one must we prefer: thoracotomy or sternotomy? Ulus Travma Acil Cerrahi Derg. 2015;21:266-70.

26. Flaris, A.N., Simms, E.R., Prat, N. et al. Clamshell Incision Versus Left Anterolateral Thoracotomy. Which One is Faster When Performing a Resuscitative Thoracotomy? The Tortoise and the Hare Revisited. World J Surg. 2015;39(5):1306-11.

27. Topal AE, Celik Y, Eren MN. Predictors of outcome in penetrating cardiac injuries. J Trauma. 2010;69:574-8. 